\title{
Author Correction: Reproducible, interactive, scalable and extensible microbiome data science using QIIME 2
}

Evan Bolyen (D), Jai Ram Rideout (D), Matthew R. Dillon (D), Nicholas A. Bokulich (D), Christian C. Abnet (iD), Gabriel A. Al-Ghalith (D), Harriet Alexander, Eric J. Alm (D), Manimozhiyan Arumugam (D), Francesco Asnicar (D), Yang Bai (D), Jordan E. Bisanz (D), Kyle Bittinger, Asker Brejnrod, Colin J. Brislawn, C. Titus Brown (iD, Benjamin J. Callahan (D), Andrés Mauricio Caraballo-Rodríguez (D), John Chase, Emily K. Cope, Ricardo Da Silva, Christian Diener (D), Pieter C. Dorrestein (D), Gavin M. Douglas, Daniel M. Durall D, Claire Duvallet (D), Christian F. Edwardson (D), Madeleine Ernst, Mehrbod Estaki, Jennifer Fouquier ID, Julia M. Gauglitz, Sean M. Gibbons (D), Deanna L. Gibson, Antonio Gonzalez, Kestrel Gorlick, Jiarong Guo, Benjamin Hillmann, Susan Holmes (D), Hannes Holste iD, Curtis Huttenhower (D), Gavin A. Huttley, Stefan Janssen (D), Alan K. Jarmusch, Lingjing Jiang, Benjamin D. Kaehler, Kyo Bin Kang (D), Christopher R. Keefe DiD, Paul Keim, Scott T. Kelley, Dan Knights, Irina Koester, Tomasz Kosciolek (D), Jorden Kreps (D), Morgan G. I. Langille, Joslynn Lee, Ruth Ley, Yong-Xin Liu (D), Erikka Loftfield (iD, Catherine Lozupone (D), Massoud Maher, Clarisse Marotz DiD, Bryan D. Martin (D), Daniel McDonald (D), Lauren J. Mclver, Alexey V. Melnik (D), Jessica L. Metcalf, Sydney C. Morgan, Jamie T. Morton, Ahmad Turan Naimey, Jose A. Navas-Molina (D), Louis Felix Nothias, Stephanie B. Orchanian (D), Talima Pearson DD, Samuel L. Peoples, Daniel Petras Di, Mary Lai Preuss, Elmar Pruesse, Lasse Buur Rasmussen, Adam Rivers (D), Michael S. Robeson IID, Patrick Rosenthal, Nicola Segata (D), Michael Shaffer, Arron Shiffer, Rashmi Sinha, Se Jin Song (D), John R. Spear, Austin D. Swafford, Luke R. Thompson (D), Pedro J. Torres, Pauline Trinh, Anupriya Tripathi, Peter J. Turnbaugh (D), Sabah Ul-Hasan, Justin J. J. van der Hooft (D), Fernando Vargas, Yoshiki Vázquez-Baeza, Emily Vogtmann (D), Max von Hippel(D), William Walters, Yunhu Wan, Mingxun Wang, Jonathan Warren (D, Kyle C. Weber, Charles H. D. Williamson, Amy D. Willis ID, Zhenjiang Zech Xu, Jesse R. Zaneveld, Yilong Zhang, Qiyun Zhu iD, Rob Knight (D) and J. Gregory Caporaso (D)

Correction to: Nature Biotechnology https://doi.org/10.1038/s41587-019-0209-9, published online 24 July 2019.

In the version of this article initially published, some reference citations were incorrect. The three references to Jupyter Notebooks should have cited Kluyver et al. instead of Gonzalez et al. The reference to Qiita should have cited Gonzalez et al. instead of Schloss et al. The reference to mothur should have cited Schloss et al. instead of McMurdie \& Holmes. The reference to phyloseq should have cited McMurdie \& Holmes instead of Huber et al. The reference to Bioconductor should have cited Huber et al. instead of Franzosa et al. And the reference to the biobakery suite should have cited Franzosa et al. instead of Kluyver et al. The errors have been corrected in the HTML and PDF versions of the article.

\section{Publisher Correction: Continuous evolution of base editors with expanded target compatibility and improved activity}

Benjamin W. Thuronyi (D), Luke W. Koblan, Jonathan M. Levy (D), Wei-Hsi Yeh, Christine Zheng, Gregory A. Newby D, Christopher Wilson (D), Mantu Bhaumik, Olga Shubina-Oleinik, Jeffrey R. Holt (D) and David R. Liu (D)

Correction to: Nature Biotechnology https://doi.org/10.1038/s41587-019-0193-0, published online 22 July 2019.

In the version of this article initially published online, both C's were underlined in the key following the blue rectangle in Figs. $4 \mathrm{~b}$, $5 \mathrm{a}, \mathrm{c}$ and $6 \mathrm{a}$. Only the second $\mathrm{C}$ should have been underlined. The error has been corrected in the print, PDF and HTML versions of this article.

Published online: 12 August 2019

https://doi.org/10.1038/s41587-019-0253-5 